\title{
Non-Gaussian noise effects in the dynamics of a short overdamped Josephson junction
}

\author{
G. Augello, D. Valentia ${ }^{\mathrm{a}}$, and B. Spagnolo \\ Dipartimento di Fisica e Tecnologie Relative, Group of Interdisciplinary Physics ${ }^{\mathrm{b}}$, Università di Palermo and CNISM-INFM, \\ Unità di Palermo, Viale delle Scienze, Edificio 18, I-90128 Palermo, Italy
}

Received 2 February 2010 / Received in final form 12 October 2010

Published online 29 November 2010 - (c) EDP Sciences, Società Italiana di Fisica, Springer-Verlag 2010

\begin{abstract}
The role of thermal and non-Gaussian noise on the dynamics of driven short overdamped Josephson junctions is studied. The mean escape time of the junction is investigated considering Gaussian, Cauchy-Lorentz and Lévy-Smirnov probability distributions of the noise signals. In these conditions we find resonant activation and the first evidence of noise enhanced stability in a metastable system in the presence of Lévy noise. For Cauchy-Lorentz noise source, trapping phenomena and power law dependence on the noise intensity are observed.
\end{abstract}

\section{Introduction}

The diffusion in overdamped Josephson junctions (JJs) is one of the most important examples of the wider nonequilibrium statistical problem of overdamped Brownian motion in tilted or randomly switching periodic potentials [1-4]. Moreover, JJs present a wide range of applications. In particular, in recent years, the JJs have been used to implement both superconducting quantum bits [5-8] and nanoscale superconducting quantum interference devices for detecting weak magnetic flux changes [9]. Josephson junctions, in fact, represent good candidates to realize solid state superconducting quantum bits (qubits) for quantum information processing. For this reason, JJs have been studied at very low temperature in devices making use of charge [10], flux [11] and phase qubits [12]. Moreover, JJs are widely used for their high sensitivity to magnetic flux changes [9].

The environment affects strongly the behavior of JJs, working both at high and low temperatures. In high temperature superconductors (HTSs) the presence of low frequency noise, whose intensity is related to the fluctuations in the bias current, temperature and magnetic field, was experimentally found [13]. In the low temperature superconductive devices it is also very difficult to avoid the influence of environment, that constitutes mainly a decoherence source for the system. In fact, a weak current noise can affect the coherence time in Josephson vortex qubits $[6,14]$.

In this framework the study of transient dynamics of Josephson junctions in the presence of noise sources is

\footnotetext{
a e-mail: valentid@gip.dft.unipa.it

b http://gip.dft.unipa.it
}

very interesting for the understanding of the interaction between these systems and the environment. In particular, the effects of noise strongly influence the current-voltage characteristic of Josephson junctions [15-18].

Recent experimental works led to the realization of overdamped Josephson junctions with non-hysteretic current voltage characteristics and high temperature stability [19-24]. These devices, realized with the possibility of tuning the internal damping with temperature, can be good candidates to measure the escape time from the metastable state, i.e. the initial superconductive state of the overdamped Josephson junction. We note that the ultrafast rapid single-flux-quantum (RSFQ) digital circuits are based on a reproduction of quantum pulses due to spasmodic changing by $2 \pi$ of the phase difference of damped Josephson junctions. The major restriction in the development of RSFQ logic circuits is given by the influence of the fluctuations [25-33], which are the main source of error when an RSFQ circuit operates at high speed. In fact, the time interval between input and output pulses of the Josephson juctions that compose the RSFQ circuit is directly connected with the average escape time from the superconductive state and depends on the noise intensity. Therefore it is important to investigate the influence of fluctuations on temporal characteristics of overdamped Josephson junctions. Finally, we note that our model could be considered as an approximation to study the dynamics in moderately damped Josephson junctions, where crossover temperature and plasma frequency, whose values are important for determining dynamical regimes, can be defined $[34,35]$.

Moreover, noise induced effects due to thermal fluctuations, such as resonant activation (RA) and noise 
enhanced stability (NES), have been theoretically predicted in overdamped JJs $[32,33]$ and, recently, experimentally revealed in underdamped JJs [36-38]. Specifically, in reference [37] the contemporaneous presence of RA and NES in underdamped JJs has been observed, finding that the average escape times can be enhanced or lowered by using different initial conditions. In reference [38], a minimum and a maximum of the average escape time as a function of the characteristic time of the temperature fluctuations have been observed. In other words, RA together with an enhancement of the life time of the metastable state can be caused not only by the oscillating barrier of the potential profile but also by the oscillating temperature.

The presence of non-Gaussian noise signals has been found experimentally in many systems. The $\alpha$-stable distributions are a very useful tool for modeling nonGaussian noise sources. A recent example of a system with non-Gaussian environmental interaction, where the noise is well modeled by an $\alpha$-stable distribution, is a wireless ad hoc network with a Poisson field of co-channel users [39]. Other interesting examples are constituted by not fully thermalized systems or, in general, systems driven away from thermal equilibrium. These can manifest physical properties related to non-Gaussian noise signals, such as large energy fluctuations with heavy-tailed probability distributions. Non-equilibrated heat reservoir can be thus considered as a source of non-Gaussian noises [40-42]. Recently, the dynamics of a JJ in the presence of non-Gaussian noise has been analyzed. The effect of non-thermal noise on the average escape time from the metastable state (superconducting state) of a currentbiased JJ, coupled with non equilibrium current fluctuations, was experimentally investigated [43,44].

The relevance of the Lévy distributions appeared in many physical, natural and social complex systems. The Lévy-type statistics, in fact, is observed in various scientific areas, where scale-invariance phenomena take place or can be suspected [45-48] (for a recent review on Lévy-flights see Ref. [49] and references there). The large amount of experimental observations of Lévy noise sources in different physical, biological and complex systems determined an increasing interest in the role of non-Gaussian noise in other research fields, such as the transient dynamics of Josephson junctions. In particular, the role of the Lévy noise in the motion of an overdamped particle in a periodic potential has recently attracted great attention [50-52]. Moreover, Lévy flights in periodic potentials have been investigated in references $[53,54]$.

The aim of this research work is to study the effects of Gaussian and non-Gaussian noise sources on the transient dynamics of point overdamped Josephson junctions. The noise simulates both the interaction of the system with the environment and the application of an external randomly fluctuating source on the system. The latter circumstance is motivated by the possibility to profit by the effects of the noise sources. We study the effects of thermal and nonthermal noise sources on the escape time of the junctions, investigating the mean lifetime of the metastable state, i.e. the superconducting state, in the presence of a periodical driving force. In this physical context, we observe resonant activation and the first evidence of noise enhanced stability in the presence of Lévy noise. A trapping phenomenon in the case of Cauchy-Lorentz noise is observed. A similar phenomenon was found in underdamped limit in reference [55].

The paper is organized as follows. In the next section the Lévy noise properties are summarized. In Section 3 the transient dynamics of short JJs is analyzed. Finally, in Section 4 we draw the conclusions.

\section{Lévy noise}

The investigation of the effects of non-Gaussian noise has been performed using $\alpha$-stable distributions as a model to generate non-Gaussian random signals [56]. Lévy processes are characterized by stationary independent increments $[57,58]$, which means that $\{L(t), t \geq 0\}$ is a Lévy process if, for every $t, \tau \geq 0$, the increment $L(t+\tau)-L(t)$ is independent of the process $\left\{L\left(t^{\prime}\right), 0 \leq t^{\prime}<t\right\}$ and follows the same law as $L(\tau)$. In particular, $L(0)=0$. The random variable $L(t)$ can be divided into the sum of an arbitrary number of independent and identically distributed random variables, as it follows from the decomposition [49]

$$
\begin{aligned}
L(t)= & L\left(\frac{t}{n}\right)+\left[L\left(\frac{2 t}{n}\right)-L\left(\frac{t}{n}\right)\right]+\ldots \\
& +\left[L\left(\frac{n t}{n}\right)-L\left(\frac{(n-1) t}{n}\right)\right] .
\end{aligned}
$$

That is, the probability distribution of $L(t)$ belongs to the class of infinitely divisible distributions (i.d.d.) [59-64]. Its second characteristics, i.e. the logarithm of characteristic function of the random variable $L(t)$ in the LévyKhinchine form [64], is

$$
\begin{aligned}
\phi(k, t) & =\ln \left\langle e^{i k L(t)}\right\rangle \\
& =\int_{-\infty}^{\infty}\left(e^{i k x}-1-i k \sin x\right) \frac{\rho(x, t)}{x^{2}} d x
\end{aligned}
$$

where $\rho(x, t)$ is the canonical measure density (with respect to the first argument) [49].

A subclass of i.d.d. is that of the stable distributions. The most general expression for the characteristic function of the random stable process $[64,65]$ is

$$
\varphi(k)=\exp \left[i k \mu-|\sigma k|^{\alpha}(1-i \beta \operatorname{sgn}(k) \Phi)\right]
$$

where $\operatorname{sgn}(k)$ is the sign function with

$$
\left\{\begin{array}{lr}
\Phi=\tan (\pi \alpha / 2), & \text { for all } \alpha \neq 1 \\
\Phi=-(2 / \pi) \log |k|, & \text { for } \alpha=1 .
\end{array}\right.
$$

Such distributions form a four-parameter family of continuous probability distributions with two shape parameters $\alpha$ and $\beta$, a scale parameter $\sigma$ and a real number $\mu$. Specifically, $\alpha(0<\alpha \leq 2)$ is the index of stability 


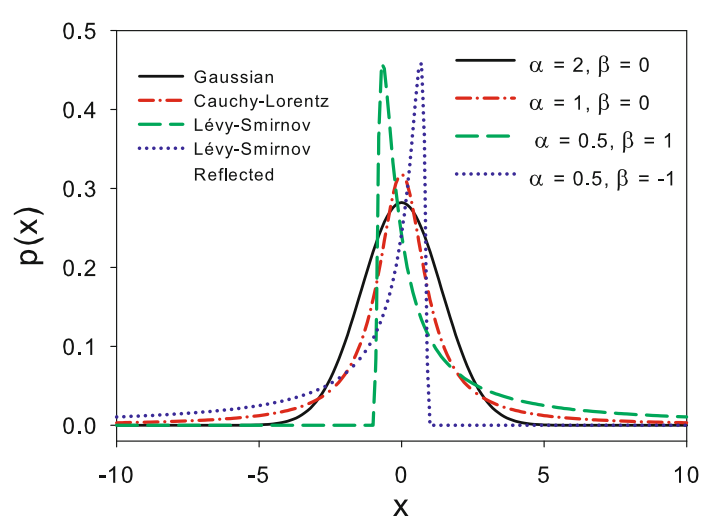

Fig. 1. (Color online) Probability density function for the four stable distributions: Gaussian (black solid line), CauchyLorentz (red dashed point line), Lévy-Smirnov (green dashed line) and Lévy-Smirnov reflected (blue dotted line). Here $\mu=0$ and $\sigma=1$.

Table 1. Stable distributions and corresponding values of the characteristic parameters.

\begin{tabular}{lcc}
\hline Distribution & $\alpha$ & $\beta$ \\
\hline Gaussian $S_{2}(\sigma, 0, \mu)$ & 2 & 0 \\
Cauchy-Lorentz $S_{1}(\sigma, 0, \mu)$ & 1 & 0 \\
Lévy-Smirnov $S_{1 / 2}(\sigma, 1, \mu)$ & $1 / 2$ & 1 \\
Lévy-Smirnov (reflected) $S_{1 / 2}(\sigma,-1, \mu)$ & $1 / 2$ & -1 \\
\hline
\end{tabular}

or characteristic exponent and indicates the asymptotic behaviour of the distribution (long-tail power law for $\alpha$ strictly less than 2$), \beta(\in[-1,1])$ is an asymmetry parameter, $\sigma$ is any positive real number which provides for $\alpha=2$ a measure of the width of the distribution, $\mu$ is any real number [65] indicating, for $\alpha>1$ and $\beta=0$, the median of the distribution. Considering Lévy distributed noise sources, $\sigma^{\alpha}$ is the noise intensity. Probability distributions for different values of $\alpha$ and $\beta$ are shown in Figure $1[65,66]$. We note that for $\beta=0$, a Lévy symmetric $\alpha$-stable distribution is obtained. By considering, for $\mu=0, \beta=0$ and $\sigma=1$, the series expansion valid for large arguments $|x| \gg 0$ [67], we obtain for large values of $x$ the following asymptotic expression characterized by a power law behaviour [65]

$$
l_{\alpha, \beta}(x ; 1,0) \sim|x|^{-(1+\alpha)}, \quad x \rightarrow \infty,
$$

which is deeply connected with the divergence of all moments $\left\langle x^{n}\right\rangle$ for $n \geq 2$ and $\alpha<2$.

An $\alpha$-stable (or Lévy) distribution is denoted by $S_{\alpha}(\sigma, \beta, \mu)$. When $\mu=0$ and $\sigma=1$ the distribution is named standard [56]. The Gaussian, Cauchy-Lorentz and Lévy-Smirnov distributions are stable, and their probability density functions are analytically known. In Table 1 the values of the characteristic parameters of these three stable distributions (shown in Fig. 1) are given. In comparison with the Gaussian case, Cauchy-Lorentz distribution is narrower in the central part, while at the extremities it goes to zero with less steepness than the Gaussian case (fat tails). The Lévy-Smirnov distribution is shown for $\beta=-1,1$. The case for $\beta=-1$ corresponds to a curve reflected with respect to the $y$-axis. The Lévy-Smirnov distribution presents a heavy tail going to zero, with a steepness lower than both the Gaussian and Cauchy-Lorentz case.

In Figures 2a, 2b the one- and two-dimensional trajectories of a particle subjected to Gaussian, CauchyLorentz and Lévy-Smirnov noise signals are shown. The trajectories are obtained considering the position of the particle subjected to a noise signal with intensity given by $\sigma^{\alpha}=2 \gamma$. Throughout the present work, different algorithms are used to simulate the Gaussian and nonGaussian Lévy noise sources $[56,66]$. In particular the time step is $(\Delta t)^{1 / \alpha}$, with $\alpha=2$ for Gaussian distribution and $0<\alpha<2$ for non-Gaussian Lévy distributions. The one-dimensional trajectory, drawn in Figure 2a and corresponding to the noise signal with Cauchy-Lorentz distribution, presents jumps followed by displacement in space with amplitude smaller than the Gaussian case. The presence of jumps is related to the heavy tails of the distribution (see Fig. 1): the probability to get high values of the random variable $x$ is greater than in the Gaussian case. Moreover, the probability to get smaller values is less than the Gaussian case and this is evident by the comparison of the central part of the Cauchy-Lorentz and Gaussian distributions (see Fig. 1). This behavior explains the limited space displacement of the trajectory corresponding to the Cauchy-Lorentz distribution for relatively low noise intensities at short times. The one-dimensional trajectory corresponding to the Lévy-Smirnov distribution shows a diffusion governed by very intense jumps. This behavior is due to the heavier tail of the Lévy-Smirnov distribution. In fact, the probability to get very high values of the random variable is greater than the Gaussian and CauchyLorentz cases. The two-dimensional trajectories provide a comparison between the different diffusion properties of the particle subjected to the three noise signals at short times. In particular, in Figure $2 \mathrm{~b}$ it is worth noting that the trajectory corresponding to the Cauchy-Lorentz distribution diffuses in space less than the Gaussian one, while the jumps of the trajectory corresponding to the LévySmirnov distribution provide a very intense spatial diffusion of the particle.

\section{Short Josephson junctions}

\subsection{Model}

The theoretical description of the dynamics of an overdamped Josephson junction is based on the resistively shunted junction (RSJ) model $[68,69]$, in which a fictitious Brownian particle is moving in a washboard potential $[70,71]$. The position of this fictitious particle represents the phase difference $\varphi$ between the superconducting wave functions on each side of the junction $[68,69]$.

Non-thermal noise sources could be also present in the system or could be applied externally to it. Therefore, the theoretical investigation of the escape from the metastable state in a JJ, out of its zero voltage state and driven by 

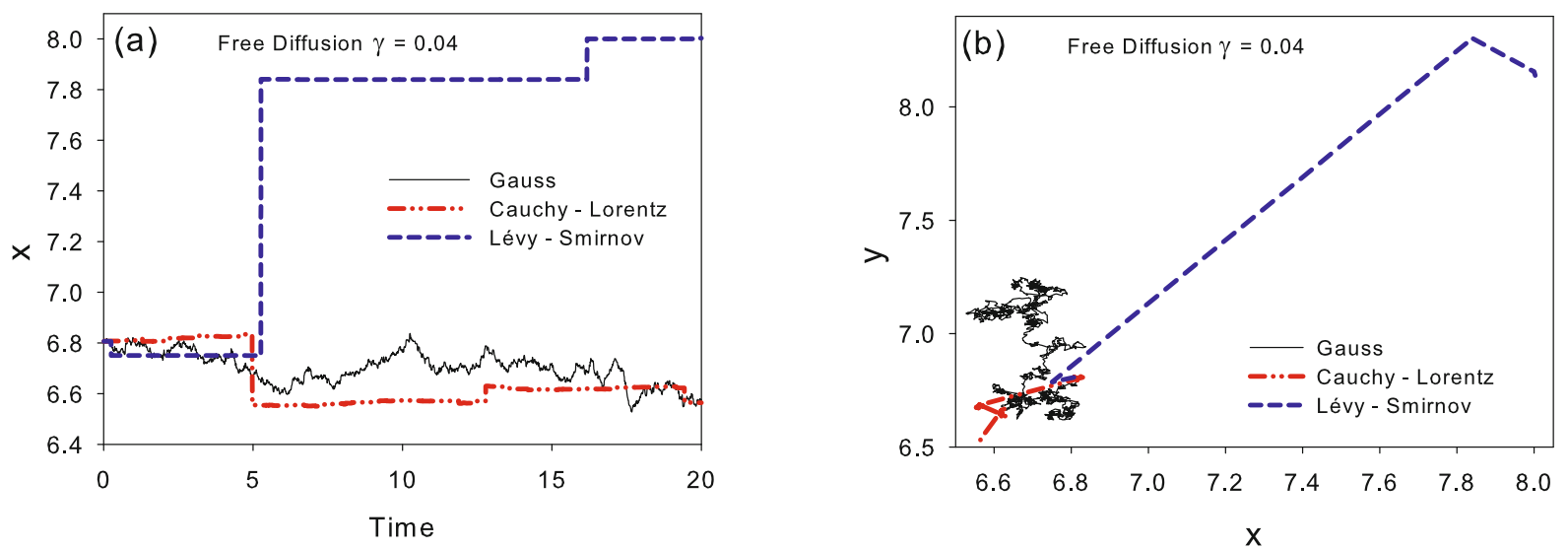

Fig. 2. (Color online) (a) One-dimensional trajectories of the free diffusion of a particle subjected to noise signals with Gaussian, Cauchy-Lorentz and Lévy-Smirnov distributions; (b) two-dimensional trajectories of the free diffusion of a particle subjected to noise signals with Gaussian, Cauchy-Lorentz and Lévy-Smirnov distributions. In both panels, the noise intensity is $\sigma^{\alpha}=2 \gamma$, with $\gamma=0.04$.

thermal as well as non-Gaussian noise, will be performed by considering the following Langevin equation for the phase dynamics

$$
\frac{d \varphi}{d t}=-\omega_{c} \frac{d U(\varphi, t)}{d \varphi}-\omega_{c}\left(i_{T N}(t)+i_{n G}(t)\right)
$$

where $i_{T N}(t)$ and $i_{n G}(t)$ are a Gaussian thermal noise and a non-Gaussian noise source, respectively. The current $i_{n G}(t)$ is a white Lévy noise such that the time integral over an increment $\Delta t, L(\Delta t)=\int_{t}^{t+\Delta t} \xi_{\alpha}(\tau) d \tau$, is a Lévy process with characteristic function given by $[50,72,73]$

$$
\phi(k, t)=\exp \left[-\Delta t|\sigma k|^{\alpha}(1-i \beta \operatorname{sgn}(k) \Phi)\right],
$$

where the parameter $\mu$ is equal to zero, $\Phi$ is given by equation (4), $\alpha$ is the Lévy index and $\sigma^{\alpha}$ is the noise intensity. The expression of the dimensionless phase potential is

$$
\begin{aligned}
\phi(k, t) & =\ln \left\langle e^{i k L(t)}\right\rangle \\
& =\int_{-\infty}^{\infty}\left(e^{i k x}-1-i k \sin x\right) \frac{\rho(x, t)}{x^{2}} d x, \\
U(\varphi, t) & =1-\cos (\varphi)+i(t) \varphi,
\end{aligned}
$$

where

$$
i(t)=i_{0}+A \sin \omega t,
$$

with $i_{0}=i_{b} / i_{c}$ the constant dimensionless bias current, $A \sin \omega t$ the driving current with dimensionless amplitude $A=i_{s} / i_{c}$ and frequency $\omega\left(i_{b}\right.$ and $i_{s}$ represent the bias current and the driving current amplitude respectively). In the following, the times and frequency will be normalized to the inverse of the characteristic frequency $1 / \omega_{c}$ and to the characteristic frequency $\omega_{c}$ of the JJ, respectively $\left(\omega_{c}=2 e R_{N} I_{c} / \hbar, i_{c}\right.$ is the critical current, $R_{N}^{-1}=G_{N}$ is the normal conductivity of the JJ, $e$ is the electron charge and $\hbar=h / 2 \pi$, with $h$ the Planck constant). The thermal fluctuations have the usual statistical properties

$$
\left\langle i_{T N}(t)\right\rangle=0, \quad\left\langle i_{T N}(t) i_{T N}(t+\tau)\right\rangle=2 \gamma \delta(\tau) / \omega_{c},
$$

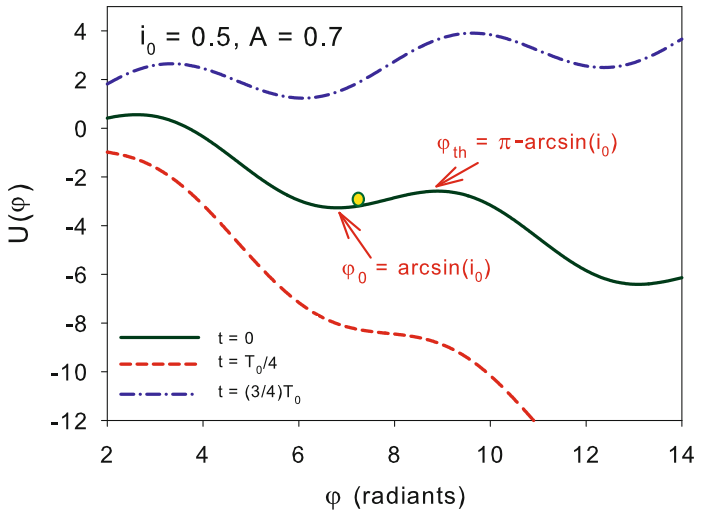

Fig. 3. (Color online) Washboard Josephson junction potential in the presence of an oscillating driving current. Screen shot of the potential profile at three different instants within one oscillation period $T_{0}: t=0, t=T_{0} / 4$ and $t=(3 / 4) T_{0}$. The arrows indicate the potential minimum corresponding to the initial condition for the fictitious particle $\left(\varphi_{0}=\arcsin i_{0}\right)$, and the potential maximum corresponding to the threshold position of the particle $\left(\varphi_{t h}=\pi-\arcsin i_{0}\right)$.

where $\gamma=\frac{2 e k T}{\hbar i_{c}}$ is the dimensionless intensity of fluctuations, $T$ is the temperature and $k$ is the Boltzmann constant.

In Figure 3 the periodic potential profile, subjected to an oscillating driving current, is shown at three different times of the period of oscillation $T_{0}$. The dynamics of the system is studied considering as initial position of the particle the zero-voltage superconductive state, corresponding to one of the minima of the potential profile ( $\varphi_{0}=\arcsin i_{0}$ in Fig. 3, with $i_{0}$ the dimensionless bias current (see Eq. 10)). When the particle is moving along the potential, the junction switches to the resistive state and a voltage develops.

The maximum, indicated in Figure 3 at $\varphi_{t h}=(\pi-$ $\left.\arcsin i_{0}\right)$, is chosen as the threshold phase value for the escape event. When the particle reaches the threshold, namely when its phase is equal or greater than $\varphi_{t h}$, the 
phase difference varies in time and the junction switches to the resistive state.

The transition from the superconductive state to the resistive state is induced on the junction by the application of a polarizing current exceeding the critical current value $i_{c}$. In the deterministic regime, at low driving frequency, the particle reaches the threshold approximately after one quarter of the period of oscillation and the escape time is $t_{\text {escape }} \approx T_{0} / 4$. This no longer occurs in the presence of noise, when the particle moves randomly along the curve. Because of the interactions with the environment, the superconductive state becomes a metastable state for the system and the transition can occur even if the polarization current is less than the critical current value. In this case, the phase difference $\varphi$ can escape from the metastable state by macroscopic quantum tunneling through the potential barrier [74] or by thermal activation (TA) over the potential barrier [75], depending on the value of the temperature $T$ with respect to the cross-over temperature defined as $T_{c o}=\hbar \omega_{p} / 2 \pi k$. Here $\omega_{p}$ is the plasma frequency, which characterizes the oscillations of the phase difference around the local minimum of the potential and $k$ is the Boltzman constant [44]. The cross-over temperature is the temperature below which the phase difference over the junction behaves quantum mechanically, the escape events occur primarily by quantum tunneling trough the barrier, and the thermal fluctuations can be neglected. For temperatures greater than $T_{c o}$ the viceversa happens. In other words we have quantum tunneling regime for $T<T_{c o}$ and thermal activation regime for $T>T_{c o}$.

The Langevin equation (6) has been integrated by using the Euler method and the $\alpha$-stable distributions of the noise signals have been numerically simulated using the Chambers-Mallows-Stuck method [56]. The time spent by the particle to reach $\varphi_{t h}$, starting from $\varphi_{0}$, under the influence of the fluctuating potential and the noise signal is the first passage time and constitutes the lifetime of the superconductive metastable state. Up to 50000 simulations of the particle trajectory have been performed to collect the corresponding first passage times. The average of these times is the mean escape time (MET) of the junction. The dimensionless bias current $i_{0}$ of the driving signal was kept under the critical current value $\left(i_{b}<i_{c}\right)$, while the values of the amplitude $A$ (Eq. (10)) have been chosen such as the particle is, during the oscillation of the driving current, in the superconductive $\left(i(t)<i_{c}\right)$ and in the resistive state $\left(i(t)>i_{c}\right)$, alternatively.

\subsection{Simultaneous action of non-Gaussian and thermal noise}

To study the simultaneous influence of thermal and non-Gaussian noise on the dynamics of a point JJ we consider in equation (6) a Lévy noise generated by a Cauchy-Lorentz distribution. The intensity of the two noise signals are indicated by $\gamma_{T N}$ and $\gamma_{\text {Cauchy-Lorentz }}$. The results, obtained by numerically solving equation (6), are displayed in Figures 4 and 5 . Here, the behavior of

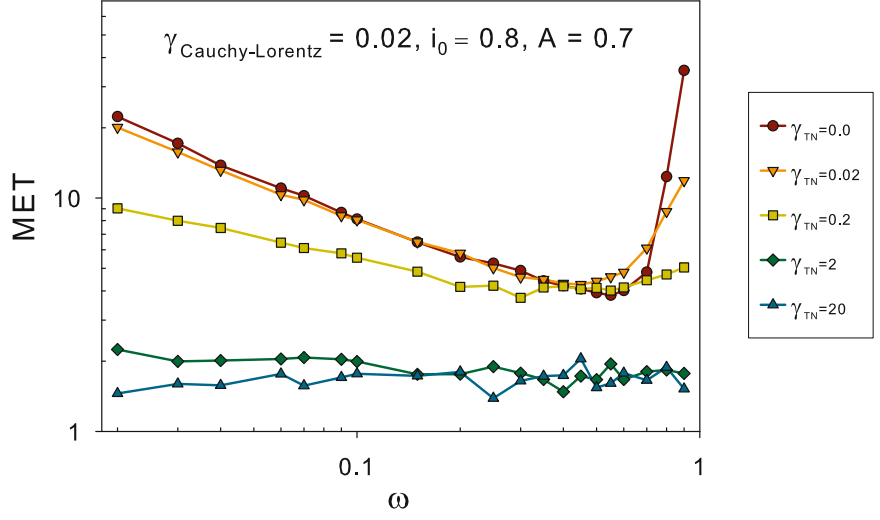

Fig. 4. (Color online) Log-Log plot of MET vs. $\omega$ for different thermal noise intensities $\gamma_{T N}$ and for fixed Lévy noise intensity $\gamma_{\text {Cauchy-Lorentz }}$. Namely, $\gamma_{T N}=0.0,0.02,0.2,2,20$, and $\gamma_{\text {Cauchy-Lorentz }}=0.02$. Here $i_{0}=0.8$ and $A=0.7$.

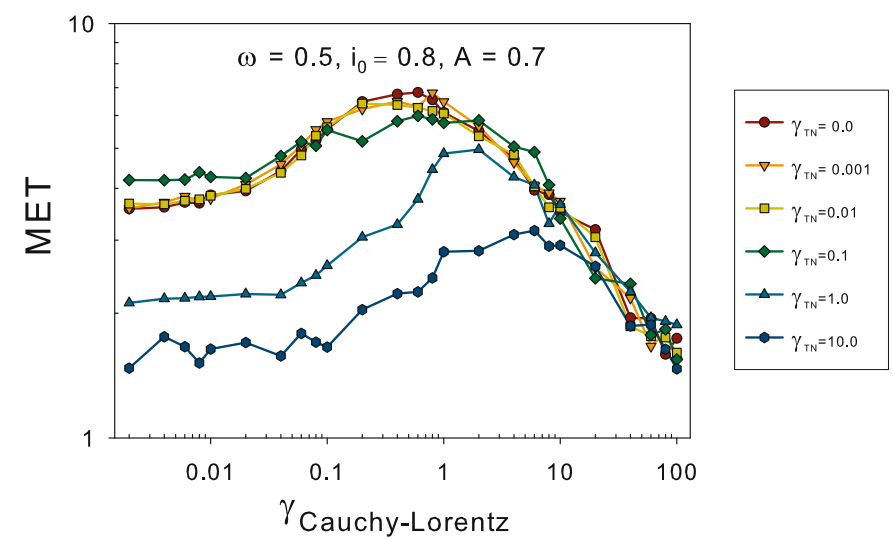

Fig. 5. (Color online) Log-Log plot of MET vs. $\gamma_{\text {Cauchy-Lorent } z}$ for different thermal noise intensities $\gamma_{T N}$. Namely, $\gamma_{T N}=$ $0.0,0.001,0.01,0.1,1.0,10.0$ and fixed frequency $\omega=0.5$. The other parameter values are as in Figure 4.

MET as a function of the driving signal frequency and the intensity of the Cauchy-Lorentz noise is shown. The curve of Figure 4 corresponding to $\gamma_{T N}=0.0$ shows a nonmonotonic behavior with a minimum, that is the signature of the RA phenomenon [76-85]. The resonant activation effect is a robust enough phenomenon to be observed also in the presence of Lévy noise sources. By increasing the thermal noise intensity $\gamma_{T N}$, the non-monotonic behavior of the MET as a function of the frequency is modified. This modification becomes evident for $\gamma_{T N} \geq 0.2$. While the position of the minimum is slightly affected by the presence of the thermal noise signal, the METs for low and high frequency values decrease. For very high frequency values, that is for very fast fluctuations of the potential profile, the mean escape time is equal to the crossing time over the average barrier "seen" by the Brownian particle [76-78]. The average barrier coincides with that obtained with the potential profile at $t=0$ (see Fig. 3) and whose height is $\Delta U=U\left(\varphi_{t h}-U\left(\varphi_{0}\right)\right) \simeq 2.1$. By using the Kramers formula for the average escape time [75], 
we obtain a lower MET by increasing the thermal noise intensity. For very low frequencies, that is for very slow fluctuations of the potential barrier, the average escape time is equal to the average of the crossing times in the highest and lowest configurations of the barrier, and the slowest process determines the value of the average escape time [76-78]. Again, an increase of the thermal noise intensity produces a decreasing of MET. By further increasing $\gamma_{T N}$, the typical RA behavior of MET vs. $\omega$ disappears because the thermal noise intensity becomes comparable with the height of the potential barrier $\gamma_{T N} \simeq \Delta U(\varphi) \simeq 2$.

The curves of Figure 5 show a non-monotonic behavior with a maximum that reveals the presence of NES effect [86-89], which is the first observation of this effect with Lévy noise. In the absence of thermal noise $\left(\gamma_{T N}=0\right.$ in Fig. 5), the behavior of the MET as a function of the noise intensity is non-monotonic with a maximum in correspondence of $\gamma_{\text {Cauchy-Lorent } z}^{\text {Max }} \simeq 0.6$. Low thermal noise signals do not affect the behavior of the NES curve, till their intensity is lower than $\gamma_{\text {Cauchy-Lorentz }}^{\text {Max }} \simeq 0.6$. When the intensity of the thermal noise exceeds this value (curves corresponding to $\gamma_{T N}=1.0$ and $\gamma_{T N}=10.0$ in Fig. 5), the thermal effects are more effective than those due to the Cauchy noise and the non-monotonic behavior is modified. The maximum of the curve decreases and it is shifted in correspondence of Cauchy noise intensities of the same order of magnitude of the thermal noise intensities. The simultaneous presence of two noise sources produces an increase of the overall noise intensity "felt" by the system. Therefore, all the MET values are lowered (see the curves for $\gamma_{T N}=1.0,10.0$ in Fig. 5). Moreover the maximum is shifted, because of the larger region of the potential profile spanned by the fictitious particle before reaching the threshold (see Figs. 3 and 5). Then, for intensities of the Cauchy noise exceeding those of the thermal noise, the curves follow the behavior obtained in the absence of thermal noise. Moreover all the curves of MET coalesce together at higher noise intensities, when the structure of the potential profile becomes irrelevant for the dynamics of the particle. The MET has a power-law dependence on the noise intensity [49]. The simultaneous presence of thermal and Cauchy noise sources in a point JJ produces different effects on the noise induced phenomena considered. At high thermal noise intensities RA disappears and the MET becomes independent on the frequency, while NES phenomenon still persists.

\subsection{Effects of non-Gaussian noise}

For low temperatures of the system (around the crossover value $\left(T \approx T_{c o}\right)$ ), we can neglect thermal fluctuations with respect to the non-Gaussian noise sources. Our starting point is now equation $(6)$, with $i_{T N}(t)=0.0$ and $i_{n G}(t)$ given by Cauchy-Lorentz, reflected Lévy-Smirnov and Gaussian distributions. The minus sign of $i_{n G}(t)$ in equation (6) indicates that the reflected Lévy-Smirnov distribution has been considered. By this way the Lévy jumps push the particle in the positive $\varphi$ direction. We note that by considering the Lévy-Smirnov distribution with $\beta=1$ (green dashed line in Fig. 1), the Brownian particle moves along the potential profile in the opposite direction with respect to the threshold phase (see Fig. 3). As a result, the MET becomes very large and diverges in the limit $\gamma \rightarrow 0$, for all values of $\omega$, as found in simulations. For this reason, the corresponding numerical results are not here shown. The curves of the MET as a function of the frequency of the driving current signal are shown in Figure 6. In each panel, the behavior of METs corresponding to noise signals with Gaussian, Cauchy-Lorentz and LévySmirnov distributions are shown. In Figures $6 \mathrm{a}-6 \mathrm{~d}$, the noise intensity increases as $\gamma=0.005$ (panel a), $\gamma=0.02$ (panel b), $\gamma=0.05$ (panel c) and $\gamma=0.5$ (panel d). The RA phenomenon is observed for all three noise sources in panels $\mathrm{a}-\mathrm{c}$ of Figure 6 , and only for Gaussian noise in panel d (see also Ref. [32] for this case). For the noise intensities of Figures $6 \mathrm{a}-6 \mathrm{c}$, the curves corresponding to the Gaussian and Cauchy-Lorentz distributions show similar behavior. Conversely, the MET values corresponding to the Lévy-Smirnov distribution, for all the frequencies and noise intensities investigated, are shorter than in the Gaussian and Cauchy-Lorentz case.

This is more evident in the position of the minima of the three different curves. For high noise intensity, $\gamma=0.5$ in Figure 6d, the non-monotonic behavior disappears for non-Gaussian noise sources. The dynamics of the system is noise-driven and the METs are independent on the driving current frequency. The METs for Gaussian and LévySmirnov noise have values shorter than in the low noise intensity case and the escape of the particle becomes faster. In addition the Gaussian case presents METs shorter than the Cauchy-Lorentz case. The one- and two-dimensional trajectories for the three noise sources of Figure 2 provide an explanation of the MET behaviors shown in Figure 6. The Lévy jumps of Lévy-Smirnov noise push the particle out of the metastable state in a very fast way. The greater is the noise intensity, the faster the escape process. Moreover, in the presence of Cauchy-Lorentz noise, the combination of the driving signal and the limited space displacement of the trajectories has the effect to increase the MET values with respect to the Gaussian case.

In order to check the robustness of the NES effect with respect to the bias current, amplitude and frequency of the driving force, we consider different values of these parameters, obtaining the behavior of the MET as a function of the noise intensity in these new conditions. The results are shown in Figures 7a and 8. Specifically, in Figure 7a the curves corresponding to the Gaussian and CauchyLorentz distributions show NES effect as in Figure 5. For very low noise intensity, namely $\gamma<10^{-3}$, all the three curves tend to the same MET value. This is the deterministic lifetime of the superconductive state, which is of the same order of magnitude of $T_{0} / 4$, with $T_{0}$ the period of the oscillating potential barrier. By increasing the noise intensity, the MET of the curve corresponding to the Lévy-Smirnov distribution decreases monotonically, because greater Lévy jumps push the particle very fast out of the superconductive state, making the MET shorter. The 

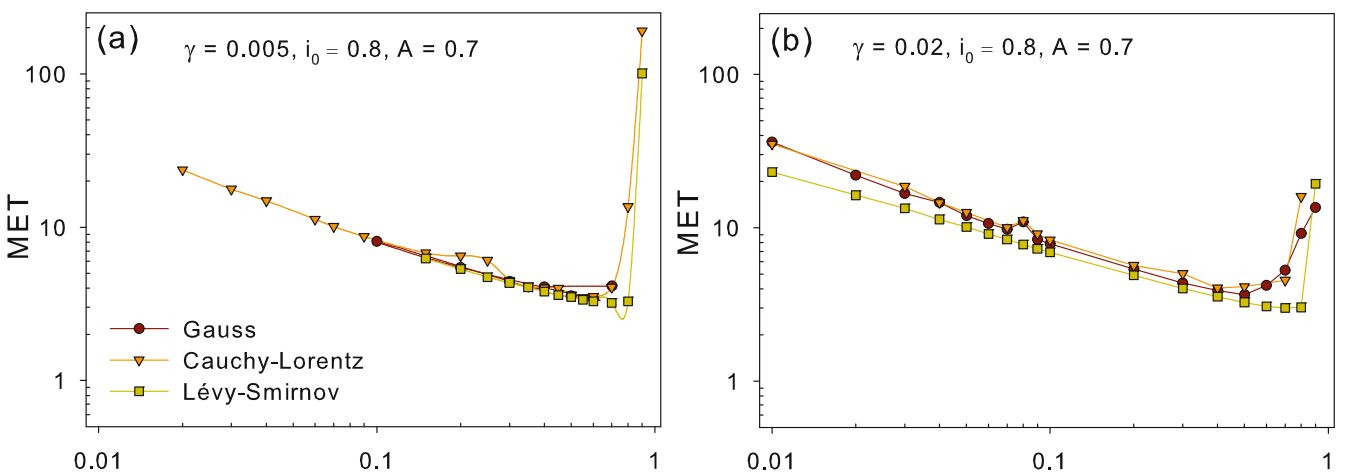

$\omega$
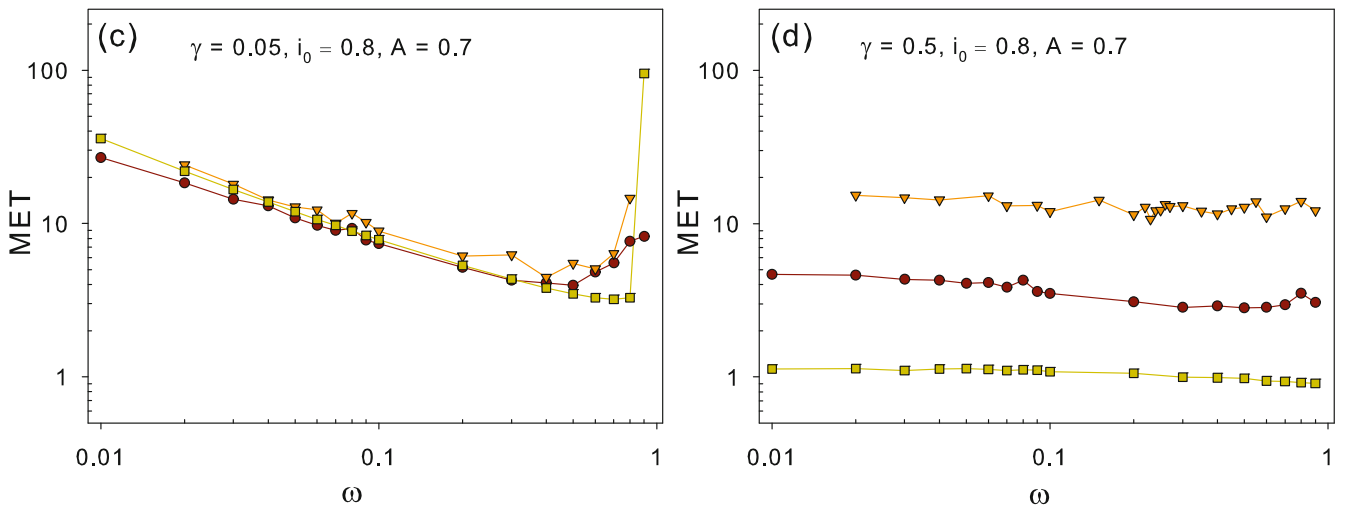

Fig. 6. (Color online) Log-Log plot of MET as a function of $\omega$ for Gaussian, Cauchy-Lorentz and Lévy-Smirnov distributions of the noise signals, for four different noise intensities. Namely, (a) $\gamma=0.005$, (b) $\gamma=0.02$, (c) $\gamma=0.05$ and (d) $\gamma=0.5$. In all graphs $i_{0}=0.8$ and $A=0.7$. The legend in panel (a) refers to all panels.
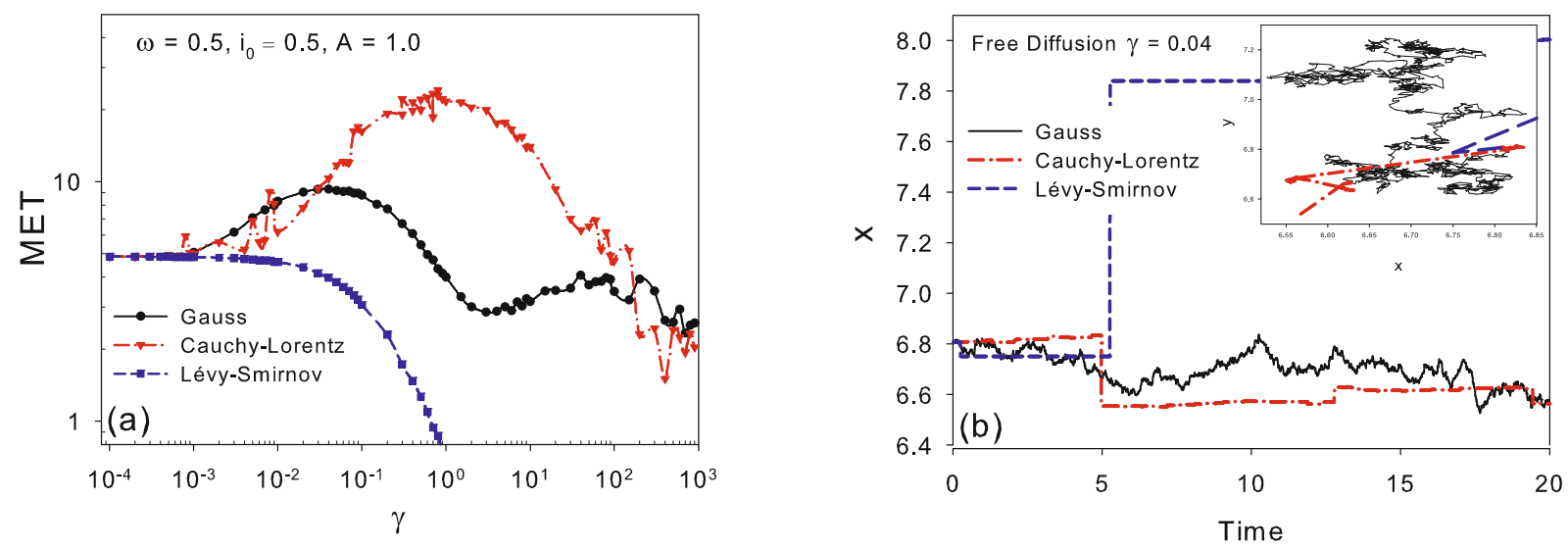

Fig. 7. (Color online) (a) Log-Log plot of MET vs. $\gamma$ for noise signals with Gaussian, Cauchy-Lorentz and Lévy-Smirnov distributions. Here $\omega=0.5, i_{0}=0.5$ and $A=1.0$. (b) One dimensional trajectories of free diffusion for a particle subjected to Gaussian, Cauchy-Lorentz and Lévy-Smirnov noise sources with $\gamma=0.04$. Inset: two-dimensional trajectories corresponding to those of the main panel.

Lévy jumps are responsible for the great diffusion power of the Lévy-Smirnov noise source (see curves of Fig. 2 shown, for convenience, also in Fig. 7b). In the range of noise intensity $10^{-3}<\gamma<1$, the curve corresponding to the Gaussian distribution presents a maximum at $\gamma \simeq 0.04$. This nonmonotonic behavior is a typical signature of the NES phenomenon [89], due to a temporary trapping effect. Here, this occurs when the mean escape time of the particle from the metastable state (superconductive state) is close to three quarter of the oscillation period of the driving force. In other words, the mean passage time to reach the maximum of the potential profile at $\varphi_{t h}$, starting from $\varphi_{0}$ (see Fig. 3), should be almost equal to $(3 / 4) T_{0}$. In fact, for $\omega=0.5$ we have $(3 / 4) T_{0}=3 \pi=9.42 \simeq M E T_{\max }=$ 9.31 (see MET curve for Gaussian noise in Fig. 7). At this time, the potential reaches its maximum reverse slope and the particle is pushed back inside the potential well, undergoing a temporary trapping phenomenon. 

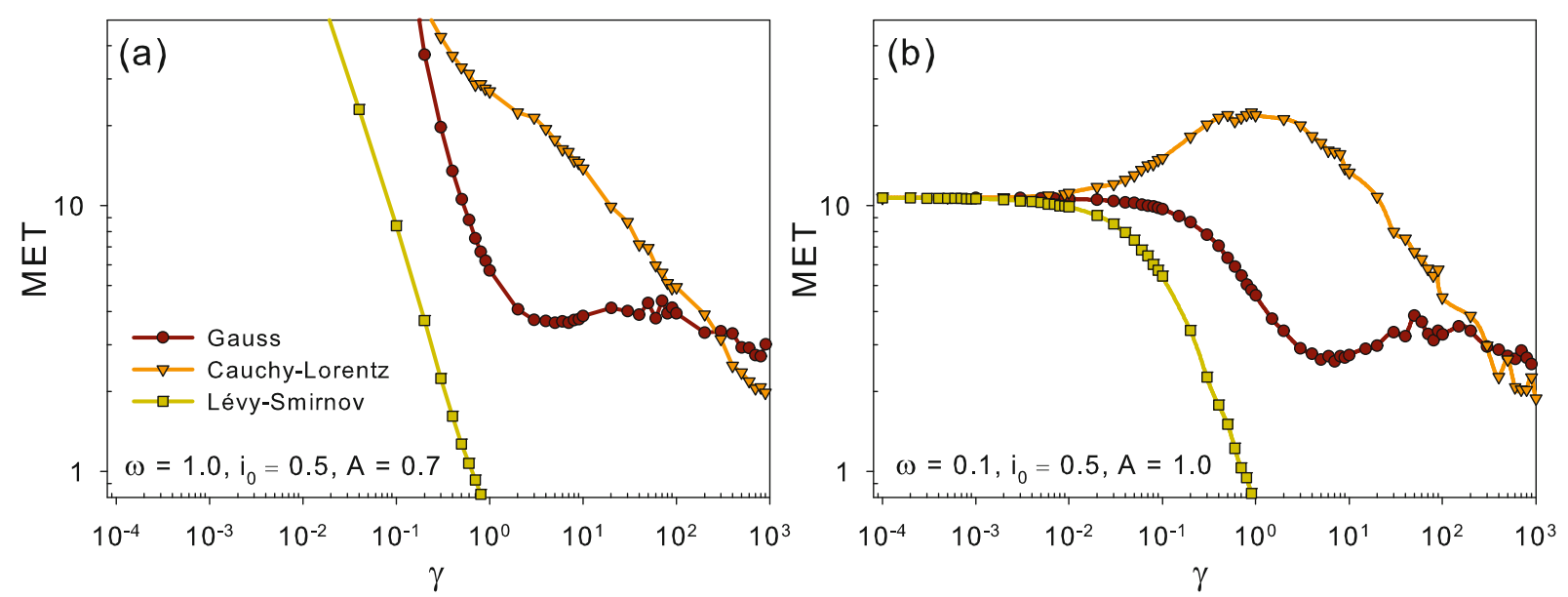

Fig. 8. (Color online) Log-Log plot of MET vs. $\gamma$ for noise signals with Gaussian, Cauchy-Lorentz and Lévy-Smirnov distributions. The values of the system parameters are: (a) $\omega=1.0, i_{0}=0.5$ and $A=0.7$; (b) $\omega=0.1, i_{0}=0.5$ and $A=1.0$.

For higher noise intensities, even in the presence of a reverse slope of the potential profile, the particle escapes from the potential well and the MET decreases till the noise intensity value $\gamma \simeq 10^{2}$. Here, the curve shows a second maximum which indicates the presence of a second weak NES effect. The MET corresponding to the second maximum results shorter than the MET of the first maximum, because of the increased noise intensity. It is also worth noting that, because of the NES effect, the particle undergoes temporary trapping phenomena preventing the escape of the junction from the initial superconductive state, even if the escape event should take place because the current $i(t)$ exceeds the critical value $i_{c}$ during the oscillation. The non-monotonic behavior is also present in the curve corresponding to the Cauchy-Lorentz distribution. The maximum of the curve is shifted towards higher noise intensity values with respect to the Gaussian case. This could be related to the fact that at short times the typical displacement of the Cauchy-Lorentz distribution is characterized by shorter steps (limited space displacement) with respect to the Gaussian case (see Fig. 2). These short times are of the same order of magnitude of the MET of the JJ analyzed. The comparison of the one-dimensional free diffusion trajectories of a particle under the influence of Gaussian and Cauchy-Lorentz noise (Fig. 7b), in correspondence of low noise intensity, namely $\gamma=0.04$, provides an useful hint to explain this behavior. In Figure $7 \mathrm{a}$, while at $\gamma=0.04$ the curve corresponding to the Gaussian noise reaches the maximum, that corresponding to the Cauchy-Lorentz noise continues to grow, and the mean lifetime of the metastable state increases with the noise intensity. From Figures $7 \mathrm{~b}$ it is evident that, even if at $t=5$ the trajectory corresponding to the Cauchy-Lorentz noise presents a jump, during the following time interval, the particle displacement is smaller than that of the Gaussian noise case. This means that at short times the particle fluctuates randomly in the potential well with an amplitude smaller than in the Gaussian case. As a consequence, the temporary trapping phenomenon is prolonged and the MET continues to grow, reaching its maximum value at a higher value of the noise intensity. For higher noise intensities the oscillation of the potential profile is no longer able to trap the particle and the MET decreases. Finally the MET vs. $\gamma$ is analyzed in the high and low frequency regime. By increasing the driving frequency, a trapping phenomenon occurs at $\omega=1$. A threshold frequency $\omega_{t h}$ exists, in fact, which does not allow the fictitious particle to move from the potential well to the next valley during one period $T_{0}$ of the driving signal. This means that for driving frequency $\omega>\omega_{t h}$, the particle is trapped within a length equal to the distance between two successive minima of the potential profile. As a consequence, the MET diverges in the limit $\gamma \rightarrow 0$. The value of the threshold frequency increases with increasing bias current and/or maximal current across the junction $[33,87,88]$. For the parameter values here considered $\left(i_{0}=0.5\right.$ and $\left.A=0.7\right)$ the threshold frequency is $\omega_{t h}=0.24$ [33]. This behavior is shown in Figure 8a for all the noise sources investigated. Specifically, for the Gaussian noise case the first maximum in the curve of Figure 7a disappears because the dynamics of the system is dominated by the very fast oscillation of the potential. We note that a minimum of MET is present for a noise intensity value of the same order of the barrier height $\left(\Delta U(\varphi) \simeq 2.1\right.$ and $\left.\gamma_{\min } \approx 3\right)[33,89]$. The second maximum, appearing for $\omega=0.5$ (Fig. 7a), persists in correspondence of $\gamma \simeq 10^{2}$. For Lévy-Smirnov noise, the MET curve decreases monotonically for higher noise intensities. Because of the intense Lévy jumps, the fictitious particle "does not see" the fine structure of the potential profile. The differences between the diffusion due to a Gaussian noise source and that caused by a Cauchy-Lorentz noise source are also present in this case, in the range of noise intensities $1<\gamma<30$. Because of the limited space displacement of the Cauchy-Lorentz noise source at short times, a slightly nonmonotonic behavior persists (very weak NES effect) with a shift, as observed at $\omega=0.5$ (see Fig. 7a).

The behavior of the MET in the low frequency regime $(\omega=0.1)$ is shown in Figure 8b. Here the amplitude of the periodic driving is $A=1.0$. For low noise intensities, 
$\gamma<10^{-2}$, all the curves tend to the deterministic lifetime of the superconductive state, which is of the same order of magnitude of $T_{0} / 4$. The MET curve for the Lévy-Smirnov noise decreases monotonically with noise intensity as in Figure 7a. For $10^{-1}<\gamma<1$ the Gaussian noise signal pushes the particle out of the potential well during the first quarter of the oscillation period and the MET is lower than $T_{0} / 4$. There is no possibility to have a temporary trapping of the particle, as observed with $\omega=0.5$ in Figure $7 \mathrm{a}$, because of the slow oscillation of the potential that permits the particle to escape before the reversing of the potential slope. Therefore, the first maximum found in Figure $7 \mathrm{a}$ in correspondence of $\gamma \simeq 0.04$ disappears. Then, for increasing noise intensity the curve shows the second maximum at $\gamma \simeq 10^{2}$, as observed for $\omega=0.5$ in Figure 7 a.

The curve corresponding to the Cauchy-Lorentz distribution shows NES effect with the same maximum at $\gamma \simeq 1$ observed at higher frequency $\omega=0.5$ (Fig. 7a). The limited diffusion due to the Cauchy-Lorentz noise allows the temporary trapping phenomenon of the particle inside the potential well, even at the very low frequency $\omega=0.1$.

\section{Conclusions}

We presented a study on the transient dynamics of an overdamped point Josephson junction in the framework of the RSJ model. We investigated the effects of Gaussian and non-Gaussian noise sources on the mean lifetime of the superconductive metastable state, by numerically solving equation (6). The resonant activation and noise enhanced stability are robust enough phenomena to be observed also in the presence of Lévy noise sources. In this paper we report on the first observation of NES effect in a metastable system with Lévy noise. For temperature greater than the cross-over value, the simultaneous presence of thermal and Cauchy noise sources in a point JJ affects differently the noise induced effects considered. At high thermal noise intensities RA disappears, while NES phenomenon still persists. Moreover, for high intensities of Cauchy-Lorentz noise, the behavior of MET vs. $\gamma$ shows a power-law dependence.

For low temperatures of the system, by neglecting thermal fluctuations, the effects of non-Gaussian noise sources on the transient dynamics of a point JJ has been analyzed. The presence of non-Gaussian noise can speed up the escape of the particle from the metastable state decreasing the MET of the junction, as observed for Lévy-Smirnov noise in the RA phenomenon. The limited diffusion of the trajectories of the Cauchy-Lorentz noise, at short times, produces MET values higher than those obtained with Gaussian noise. By a suitable choice of the parameter values that characterize the system, such as bias current, amplitude and frequency of the periodic driving signal, temporary and permanent trapping phenomena can be observed. This produces an increase of the mean lifetime of the metastable state and therefore of the MET. The knowledge of the effects of different non-Gaussian noise sources on the dynamics of a Josephson junction is useful to better understand and control the response of superconductive devices based on the Josephson junction. In particular, the results of our numerical investigation can provide useful information before the realization of experimental setups which are, sometimes, very expensive.

Authors acknowledge the financial support by MIUR and CNISM-INFM.

\section{References}

1. B. Lindner, M. Kostur, L. Schimansky-Geier, Fluc. Noise Lett. 1, R25 (2001)

2. P. Reimann, C. Van den Broeck, H. Linke, P. Hänggi, J.M. Rubi, A. Pérez-Madrid, Phys. Rev. Lett. 87, 010602 (2001)

3. P. Reimann, C. Van den Broeck, H. Linke, P. Hänggi, J.M. Rubi, A. Pérez-Madrid, Phys. Rev. E 65, 031104 (2002)

4. A.A. Dubkov, B. Spagnolo, Phys. Rev. E 72, 041104 (2005)

5. G. Wendin, V.S. Shumeiko, Low Temp. Phys. 33, 724 (2007)

6. J.H. Kim, R.P. Dhungana, K.S. Park, Phys. Rev. B 73, 214506 (2006)

7. A.B. Zorin, E.M. Tolkacheva, M.I. Khabipov, F.-I. Buchholz, J. Niemeyer, Phys. Rev. B 74, 014508 (2006)

8. A.J. Berkley, H. Xu, M.A. Gubrud, R.C. Ramos, J.R. Anderson, C.J. Lobb, F.C. Wellstood, Phys. Rev. B 68, $060502(2003)$

9. C.H. Wu, Y.T. Chou, W.C. Kuo, J.H. Chen, L.M. Wang, J.C. Chen, K.L. Chen, U.C. Sou, H.C. Yang, J.T. Jeng, Nanotechnology 19, 315304 (2008)

10. Y. Nakamura, Yu. A. Pashkin, J.S. Tsai, Nature 398, 786 (1999)

11. J.R. Friedman, V. Patel, W. Chen, S.K. Tolpygo, J.E. Lukens, Nature 406, 43 (2000)

12. J.M. Martinis, S. Nam, J. Aumentado, C. Urbina, Phys. Rev. Lett. 89, 117901 (2002)

13. L. Hao, J.C. Macfarlane, Physica C: Superconductivity 292, 315 (1997)

14. H. Xu, A.J. Berkley, R.C. Ramos, M.A. Gubrud, P.R. Johnson, F.W. Strauch, A.J. Dragt, J.R. Anderson, C.J. Lobb, F.C. Wellstood, Phys. Rev. B 71, 064512 (2005)

15. C.M. Falco, W.H. Parker, S.E. Trullinger, P.K. Hansma, Phys. Rev. B 10, 1865 (1974)

16. J.T. Peltonen, A.V. Timofeev, M. Meschke, J.P. Pekola, J. Low Temp. Phys. 146, 135 (2006)

17. A. Marx, U. Fath, W. Ludwig, R. Gross, T. Amrein, Phys. Rev. B 51, 6735 (1995)

18. A. Marx, U. Fath, L. Alff, R. Gross, Appl. Phys. Lett. 67, 1929 (1995)

19. V. Lacquaniti, C. Cagliero, S. Maggi, R. Steni, Appl. Phys. Lett. 86, 042501 (2005)

20. V. Lacquaniti, D. Andreone, S. Maggi, R. Rocci, A. Sosso, R. Steni, Physica C 435, 99 (2006)

21. V. Lacquaniti, D. Andreone, N. De Leo, M. Fretto, S. Maggi, A. Sosso, M. Belogolovskii, IEEE Trans. Appl. Supercond. 17, 609 (2007)

22. V. Lacquaniti, N. De Leo, M. Fretto, S. Maggi, A. Sosso, Appl. Phys. Lett. 91, 252505 (2007)

23. V. Lacquaniti, D. Andreone, C. Cassiago, N. De Leo, M. Fretto, S. Maggi, A. Sosso, M. Belogolovskii, J. Phys. Conf. Ser. 97, 012174 (2008) 
24. P. Febvre, D. Bouis, N. De Leo, M. Fretto, A. Sosso, V. Lacquaniti, J. Appl. Phys. 107, 103927 (2010)

25. A.V. Rylyakov, K.K. Likharev, IEEE Trans. Appl. Supercond. 9, 3539 (1999)

26. K.K. Likharev, V.K. Semenov, IEEE Trans. Appl. Supercond. 1, 3 (1991)

27. T. Filippov, M. Znosko, Supercond. Sci. Tecnol. 12, 776 (1999)

28. V.M. Buchstaber, O.V. Karpov, S.I. Tertychnyi, J. Exp. Theor. Phys. 97, 624 (2003)

29. K. Fujiwara et al., IEEE Trans. Appl. Supercond. 15, 427 (2005)

30. H. Terai, Z. Wang, Y. Hishimoto, S. Yorozu, A. Fujimaki, N. Yoshikawa, Appl. Phys. Lett. 84, 2133 (2004)

31. A.V. Gordeeva, A.L. Pankratov, J. Appl. Phys. 103, 103913 (2008)

32. A.L. Pankratov, B. Spagnolo, Phys. Rev. Lett. 93, 177001 (2004)

33. A.V. Gordeeva, A.L. Pankratov, B. Spagnolo, Int. J. Bifurc. Chaos 18, 2825 (2008)

34. J.C. Fenton, P.A. Warburton, J. Phys. Conf. Ser. 150, $052052(2009)$

35. J.C. Fenton, P.A. Warburton, Phys. Rev. B 78, 054526 (2008)

36. Y. Yu, S. Han, Phys. Rev. Lett. 91, 127003 (2003)

37. G. Sun, N. Dong, G. Mao, J. Chen, W. Xu, Z. Ji, L. Kang, P. Wu, Y. Yu, D. Xing, Phys. Rev. E 75, 021107 (2007)

38. C. Pan, X. Tan, Y. Yu, G. Sun, L. Kang, W. Xu, J. Chen, P. Wu, Phys. Rev. E 79, R030104 (2009)

39. M.R. Souryal, E.G. Larsson, B. Peric, B.R. Vojcic, IEEE Trans. Sign. Proc. 56, 266 (2008)

40. B. Dybiec, E. Gudowska-Nowak, Phys. Rev. E 69, 016105 (2004)

41. E.W. Montroll, M.F. Shlesinger, in Nonequilibrium Phenomena II: From Stochastics to Hydrodynamics, edited by J.L. Lebowitz, E.W. Montroll (North-Holland, Amsterdam, 1984)

42. M.F. Shlesinger, G.M. Zaslavsky, U. Frisch, Levy Flights and Related Topics in Physics (Springer-Verlag, Berlin, 1995)

43. J.T. Peltonen, A.V. Timofeev, M. Meschke, T.T. Heikkilä, J.P. Pekola, Physica E 40, 111 (2007)

44. B. Huard, H. Pothier, N.O. Birge, D. Esteve, X. Waintal, J. Ankerhold, Ann. Phys. 16, 736 (2007)

45. A.V. Chechkin, V. Yu. Gonchar, J. Klafter, R. Metzler, Adv. Chem. Phys. 133, 439 (2006)

46. R. Metzler, J. Klafter, Phys. Rep. 339, 1 (2000)

47. V.V. Uchaikin, Physics-Uspekhi 46, 821 (2003)

48. A.A. Dubkov, A. La Cognata, B. Spagnolo, J. Stat. Mech.: Theory Exp. P01002 (2009)

49. A.A. Dubkov, B. Spagnolo, V.V. Uchaikin, Int. J. Bifurc. Chaos 18, 2649 (2008)

50. D. del-Castillo-Negrete, V.Yu. Gonchar, A.V. Chechkin, Physica A 387, 6693 (2008)

51. B. Dybiec, E. Gudowska-Nowak, I.M. Sokolov, Phys. Rev. E 78, 011117 (2008)

52. B.Q. Ai, Y.F. He, J. Stat. Mech.-Theory E. P04010 (2010)

53. D. Brockmann, T. Geisel, Phys. Rev. Lett. 90, 170601 (2003)

54. A.A. Greenenko, A.V. Chechkin, N.F. Shulga, Phys. Lett. A 324, $82(2004)$

55. A.I. Shushin, Chem. Phys. 370, 244 (2010)

56. A. Weron, Stat. Prob. Lett. 28, 165 (1996)
57. J. Bertoin, Lévy Processes (Cambridge University Press, Cambridge, 1996)

58. K.I. Sato, Lévy Processes and Infinitely Divisible Distributions (Cambridge University Press, Cambridge, 1999)

59. B.V. Gnedenko, A.N. Kolmogorov, Limit Distributions for Sums of Independent Random Variables (Addison-Wesley, Cambridge, MA, 1954)

60. B. de Finetti, Rendiconti della R. Accademia Nazionale dei Lincei (Ser. VI) 10, 325 (1929)

61. B. de Finetti, Theory of Probability (Wiley, New York, 1975), Vols. 1, 2

62. A. Khintchine, P. Lévy, Comptes Rendus 202, 374 (1936)

63. A. Ya. Khintchine, Limit Distributions for the Sum of Independent Random Variables (O.N.T.I., Moscow, 1938) [in Russian]

64. W. Feller, An Introduction to Probability Theory and its Applications (John Wiley \& Sons, Inc., New York, 1971), Vol. 2

65. R.N. Mantegna, H.E. Stanley, An Introduction to Econophysics. Correlations and Complexity in Finance (Cambridge University Press, Cambridge, 2000)

66. J.P. Nolan, Stable Distributions: Models for Heavy-Tailed Data (Birkhauser-Springer, Berlin, 2002)

67. H. Bergström, Ark. Mathematicae II 18, 375 (1952)

68. A. Barone, G. Paternò, Physics and Application of the Josephson Effect (Wiley, New York, 1982)

69. K.K. Likharev, Rev. Mod. Phys. 51, 101 (1979)

70. Y. Yu, S. Han, X. Chu, S. I. Chu, Z. Wang, Science 296, $889(2002)$

71. V.I. Mel'nikov, Phys. Rep. 209, 1 (1991)

72. G. Samorodnitsky, M.S. Taqqu, Stable non-Gaussian Random Processes (Chapman \& Hall, New York, 1994), p. 619

73. A.V. Chechkin, V.Yu. Gonchar, J. Klafter, R. Metzler, Europhys. Lett. 72, 348 (2005)

74. U. Weiss, Quantum Dissipative Systems (World Scientific, Singapore, 1999)

75. P. Hänggi, P. Talkner, M. Borkovec, Rev. Mod. Phys. 62, $251(1990)$

76. C.R. Doering, J.C. Gadoua, Phys. Rev. Lett. 69, 2318 (1992)

77. R.N. Mantegna, B. Spagnolo, Phys. Rev. Lett. 84, 3025 (2000)

78. R.N. Mantegna, B. Spagnolo, J. Phys. IV (France) 8, 247 (1998)

79. P. Pechukas, P. Hänggi, Phys. Rev. Lett. 73, 2772 (1994)

80. M. Marchi, F. Marchesoni, L. Gammaitoni, E. MenichellaSaetta, S. Santucci, Phys. Rev. E 54, 3479 (1996)

81. J. Iwaniszewski, I.K. Kaufman, P.V.E. McClintock, A.J. McKane, Phys. Rev. E 61, 1170 (2000)

82. M. Boguñá, J.M. Porra, J. Masoliver, K. Lindenberg, Phys. Rev. E 57, 3990 (1998)

83. B. Dybiec, E. Gudowska-Nowak, Phys. Rev. E 66, 026123 (2002)

84. B. Dybiec, E. Gudowska-Nowak, J. Stat. Mech.-Theory E. P05004 (2009)

85. V.N. Chizhevsky, Phys. Rev. E 80, 061139 (2009)

86. R.N. Mantegna, B. Spagnolo, Phys. Rev. Lett. 76, 563 (1996)

87. N. Agudov, B. Spagnolo, Phys. Rev. E 64, R035102 (2001)

88. A.A. Dubkov, N.V. Agudov, B. Spagnolo, Phys. Rev. E 69, $061103(2004)$

89. A. Fiasconaro, B. Spagnolo, S. Boccaletti, Phys. Rev. E. 72, $061110(2005)$ 